Research Article

Oopen Access

CrossMark

\title{
Effectivity of blood flow restriction training for gains in strength and trophism in patients with $A C L$ injuries
}

\begin{abstract}
Objectives: The aim of this study is to compare the effectiveness of blood flow restriction training (BFR) against traditional training, to increase strength and muscle mass in patients with a history of ACL surgery.
\end{abstract}

Selection criteria: Men between 20-30 years old, who have had an ACL surgery with more than 3 months but no more than 3 years evolution since surgery, not doing any other physiotherapy treatment or sports activity, contraindications for BFR have been cleared before screening process. (Deep-vein thrombosis, pregnancy, varicose veins, high blood pressure, and cardiac disease)

Methodology: 6 weeks of BFR training were applied with low load resistance training $(30 \% 1 \mathrm{RM})$ for quadriceps and hamstrings muscles using BFR, controlled pressure at $150 \mathrm{mmhg}$ in the proximal thigh for no more than 30 minutes. 1 group of 5 people were assigned to a low load resistance training with $30 \% 1 \mathrm{RM}$ using BFR (experiment group) for 6 weeks, while another group of 5 people was assigned the same exercises for 6 weeks but with a high load (70\% 1RM) resistance training without BFR (control group).

Three methods of measurement were applied before and after 6 weeks of training using the following tools:

1) dynamometer (measure strength in $\mathrm{Kg} / \mathrm{Sec} 2$ during isometric quadriceps and hamstrings tests.

2) measure tape: hypertrophy was measured in the thigh at 10 and $20 \mathrm{~cm}$ above patella using this tape in international metric scale $(\mathrm{cm})$.

3) 1 RM (maximal strength repetition) test were done for leg extension and femoral curl in order to select the initial working weight in each and also to evaluate the final gains in strength after 6 weeks of training.

Results: All the participants from the BFR group had increases in dynamometer test with an average of $8.8 \mathrm{kgs} / \mathrm{s} 2$ quadriceps and $7.4 \mathrm{Kg} / \mathrm{s} 2$ hamstrings, compared with an average of $3.1 \mathrm{~kg} / \mathrm{s} 2$ quadriceps, $2.9 \mathrm{~kg} / \mathrm{s} 2$ hamstrings in control group.

All participants in BFR group had increases in limb mass volume with an average of $1.54 \mathrm{~cm}$ at $10 \mathrm{~cm}$ above patella and $1.7 \mathrm{~cm}$ at $20 \mathrm{~cm}$ above the patella, compared with the control group who obtained an average of $1.94 \mathrm{~cm} 10 \mathrm{~cm}$ above patella and $1.82 \mathrm{~cm}$ $20 \mathrm{~cm}$ above the patella.

Finally, an average increase of weight in the $1 \mathrm{RM}$ test of $25.44 \mathrm{lb}$ for leg extension and $25.00 \mathrm{lb}$ for femoral curl muscles. Compared with the control group (NO BFR) with an average of $18.7 \mathrm{lb}$ in leg extension and $22.5 \mathrm{lb}$ in femoral curl.

Discussion: the results of this study show that using a low load resistance training in combination with BFR improved the strength and muscle mass of individuals after ACL reconstruction with similar or better results compared to a traditional high load resistance training, secondary changes, like decrease of pain level and decrease in instability sensation were found in BFR group.

Keywords: blood Flow restriction training, strength training, hypertrophy training, ACL injuries
Volume 10 Issue 6 - 2018

\section{Fernandez Reyes Alejandra Marissa}

Universidad del Futbol y Ciencias del Deporte (UFD), México

Correspondence: Fernandez Reyes Alejandra Marissa, Universidad del Futbol y Ciencias del Deporte (UFD), México, Email marissaf_r@hotmail.com

Received: November 21, 2018 | Published: November 27, 2018

\section{Introduction}

Blood flow restriction (BFR) training has grown to become a common training method and technique for strength training. BFR is associated with muscle mass, endurance, and muscle oxygenation. Research has shown that BFR training can increase strength gains and support muscle hypertrophy. ${ }^{1}$ Blood flow restriction (BFR) training is a novel training strategy that involves the use of cuffs or wraps placed proximally around a limb, with the aim of maintaining arterial inflow while occluding venous return during exercise. ${ }^{2}$

BFR involves decreasing blood flow to working muscles with the 
aim of promoting hypertrophy and prevent the atrophy of muscles. BFR training is typically used during resistance exercise or can be done using compression devices solely while walking, cycling or another physical activity. These devices are similar to blood pressure cuffs, and they apply pressure high enough to occlude $50-80 \%$ of blood flow to the muscles where the change is required. ${ }^{3}$

The applications of BFR are numerous and one of the most common applications in the field of Physical therapy in recent years is post-operative recovery for Anterior Cruciate Ligament (ACL) surgical procedures. One of the biggest challenges in ACL rehab is regaining adequate strength in the knee which was operated. Even as long as a year after the operation, several patients still report significant strength deficits compared with the non-surgical leg. Traditional early post-operation rehab consists of swelling control, ROM, muscle activation, gait training, and proprioceptive exercises. Unfortunately for many persons, the leg continues to atrophy and remain in a state of anabolic resistance occasioned by the trauma of the surgical procedure and subsequent relative or literal immobilization. These early postoperation treatments and exercises while necessary, don't do enough to help the muscle hypertrophy. Traditional resistance training guides which recommend lifting loads greater than 65 percent of a one-rep max over 3-4 months to regain this lost strength. With traditional resistance training, the athlete is sometimes unable to perform this routine due to severe injuries and pain. In many occasions, it is unrealistic and potentially dangerous. ${ }^{4}$

However, with BFR, the physiotherapist may be able to improve an injured athlete's muscle protein synthesis to a positive state without compromising vulnerable joints or soft tissue. BFR can be introduced as early as a few days post-op and can stop anabolic resistance by initiating protein synthesis, which results in muscle hypertrophy even with loads of $20 \%$ of 1 RM and lower. This happens because the tourniquet creates a hypoxic environment which causes the recruitment of type 2 fibers despite the load being more appropriate for type 1 . Type 1 muscle fibers require oxygen and are important for low load exercises and endurance training. While exercising under BFR, the stored oxygen gets depleted, and the remaining reps are carried out by two muscle fibers. Type 2 muscle fibers are responsible for power and speed, and their byproduct is lactate. The cuff restricts the venomous return flow and holds the lactate in the limb which initiates a systemic response that causes the release of growth hormone by the pituitary gland. Growth hormone is responsible for the synthesis of collagen which stimulates the healing of muscle, tendon, ligament, cartilage, and bone. ${ }^{5}$

BFR works by first securing a pneumatic tourniquet (just like those used during surgery) at the most proximal point of an injured arm or leg. Vascular flow is then detected with the use of a Dopplerlike system to determine the necessary occlusion pressure of the limb which is defined as the amount of pressure required to eliminate blood flow to the limb. Eighty percent pressure in the lower extremities demonstrates the highest muscle recruitment while $40-50 \%$ percent in the upper extremities results in similar results.

Wilson et al.,6 found that in practical blood flow restriction (where wraps are used to apply pressure), a perceived wrap tightness of 7 out of 10 results in complete venous, but not arterial, occlusion. This is consistent with the stated aim of blood flow restriction training to maintain arterial inflow while occluding venous return during exercise. The effects of BFR on ACL post-surgery recovery has been researched upon (Owens Recovery Science). It was discovered that there were significant muscle gains in those persons which used a combination of low-load resistance muscular training with moderate restriction of blood flow after ACL reconstruction. From research, only the BFR cohort demonstrated a significant increase in pre to post knee extensor muscle size. Going from a ratio of 91 (injured/ uninjured) pre-surgery to 101 post-BFR intervention. There was no significant difference in the knee flexors/adductors. Also, the BFR group was able to maintain significantly more knee extension strength compared with the non-BFR group and the BFR group also was able to maintain significantly more knee flexion strength compared with the non-BFR group. ${ }^{7}$

Although the exact mechanism behind the gains gotten with BFR training is not completely understood, several theories have been presented. One common hypothesis is the recruitment of larger, fasttwitch motor units during the hypoxic state created with the tourniquet. Several papers which agree with this idea have demonstrated higher intramuscular electromyographic signal output during exercises under vascular occlusion compared with low-load training without a tourniquet. Another hypothesis is that as the muscles utilize the anaerobic pathway in the process of resistance training with BFR, its metabolic accumulation may trigger hypertrophic changes. This evidence was seen in a 2014 study of Clinical Physiology and Functional Imaging which compared the accumulation of substances such as lactate between BFR, high-intensity training, and standard low-load training. The BFR group showed a significant rise in lactate and similar levels of metabolic stress as the HIT group. The systematic response from this accumulation of metabolite with BFR has been shown to include significant increases in substances such as insulinlike growth factor and myogenic stem cells.

A third theory is that muscle pump effect seen after tourniquet training might play a role in hypertrophy gains. BFR results in muscle swelling and plasma volume shift which can help augment muscle size by activating the protein synthesis pathway MTORC1. Other studies agree with these theories by demonstrating the ability of occlusion alone to mitigate atrophy compared with controls. The cellular swelling created by a tourniquet was as a result of a lack of exercise which was enough to induce muscle protein synthesis. BFR studies concluded that occluding blood flow in veins may cause several problems because venous blood flow occlusion may prevent blood cycle from being completed and this may result in heart failure and arterial stiffness. So, the best option is to define blood flow restriction as blood flow moderation training. In this context, BFR has been shown to have several benefits such as improving sprint time compared with low-intensity sprint interval training, increased rectus femoris muscle thickness and decreased the level of muscle damage. ${ }^{8}$

\section{Methods}

Participants and recruitment: 20 people were recruited via an open invitation to participate in this study for strength training after ACL injuries, ten subjects were excluded because they did not meet the inclusion criteria.

Randomization: Randomization was performed, using an alternating envelope system. Upon presenting at the study site, evaluators assigned ten envelopes whose were five BFR and five no BFR training, patients randomly selected those envelopes before starting the first evaluation.

Assesment procedure: one PT screened all patients without the knowledge of in which group they would be assigned; ten individuals were evaluated with the same tools, dynamometer isometric strength with a 4 seconds contraction duration for quadriceps and hamstrings muscles, thigh volume 10 and $20 \mathrm{~cm}$ above the patella. 
Reliability: All three people collecting data and supervising study were physiotherapist working in an Orthopedic outpatient clinic located in Baja California, Mexico. Each PT was educated on the aims of the study, measurements tools used, completion of forms, and delivery of instructions for training before performing the evaluation and intervention. To ensure uniform application of the training the same PT supervised both groups at the training time, another one did the before and after evaluations and the last one interpreted the results.

Intervention: All patients received the same instructions about exercise routine: 3 series of 10 repetitions of leg extension training and 3 series of 10 repetitions of femoral curl training.

They did this routine 3 days a week for 6 weeks using the 1RM percentage that was allocated since the beginning, the experimental group (5 subjects) received the allocated intervention using BFR training and the control group (5 subjects) received the allocated intervention using general strength training without BFR. ${ }^{9-16}$

\section{Outcome measures}

Blood flow restriction group (experimental)

Table 1-3

Traditional resistance training group (control)

Table 4-6

Table I comparison between first evaluation and second evaluation after 6 weeks of BFR training (dynamometer measurements)) (5 subjects)

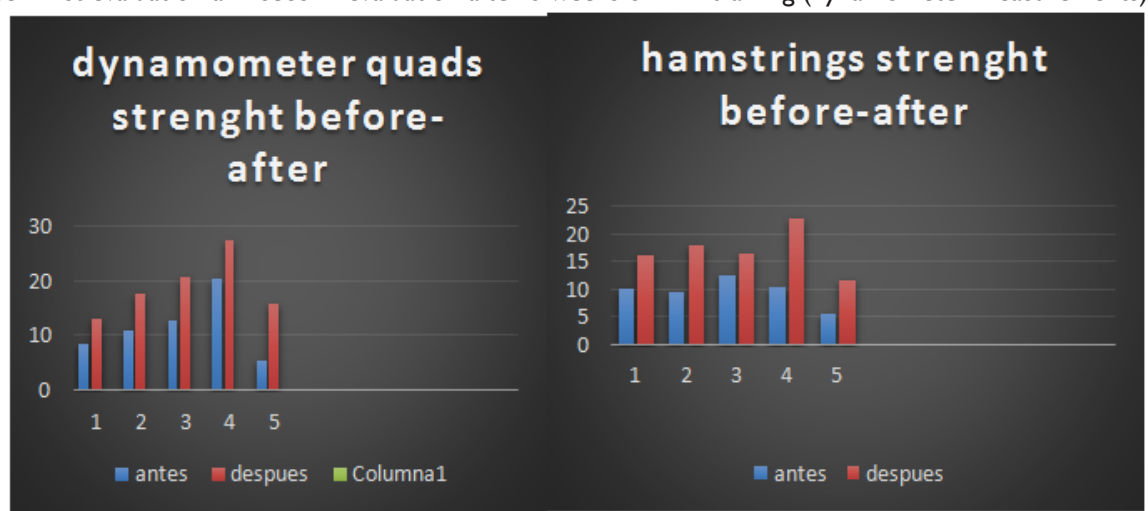

$8.8 \mathrm{~kg} / \mathrm{s} 2$. average increase

$7.4 \mathrm{Kg} / \mathrm{s} 2$ average increase

Table 2 Comparison between first evaluation and second evaluation after 6 weeks BFR training (volumetric measurement with metric tape in $\mathrm{cm}$ )



$1.54 \mathrm{~cm}$ average increase

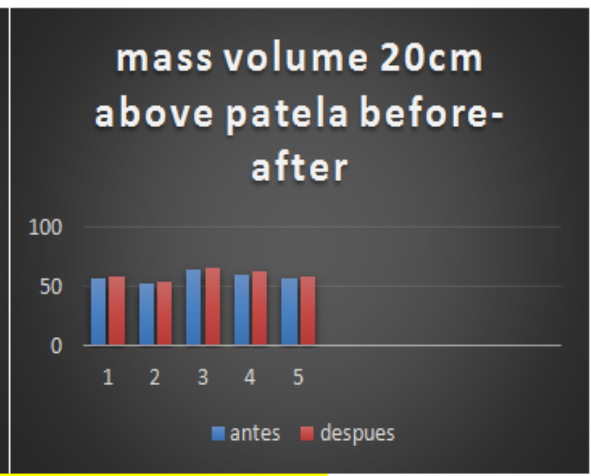

$1.7 \mathrm{~cm}$ average increase

Table 3 Comparison between first evaluation and after 6 weeks of BFR training (IRM evaluation test in quadriceps and hamstrings muscles)

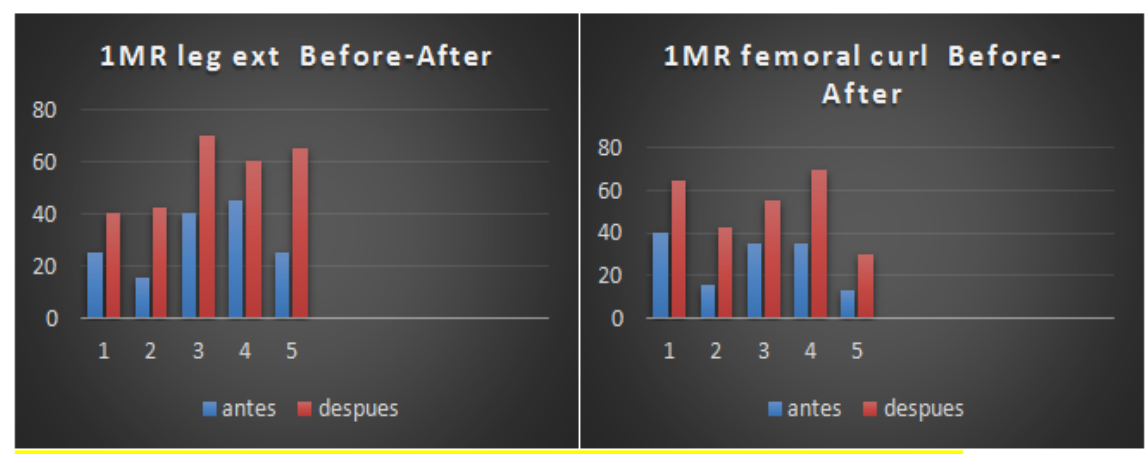

$25.44 \mathrm{lb}$ average increase in $1 \mathrm{RM}$

$25 \mathrm{lb}$ average increase in $1 \mathrm{RM}$ 
Table 4 Comparison between first evaluation and after 6 weeks of traditional resistance training (dynamometer measurements)

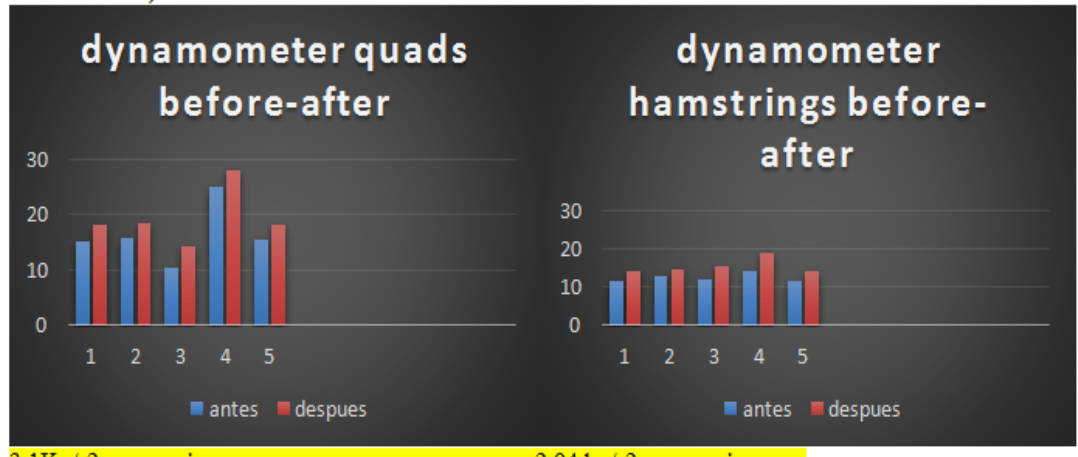

$3.1 \mathrm{Kg} / \mathrm{s} 2$ average increase

$2.94 \mathrm{~kg} / \mathrm{s} 2$ average increase

Table 5 Comparison between first evaluation and after 6 weeks of traditional resistance training (volumetric measurement with metric tape in $\mathrm{cm}$ )

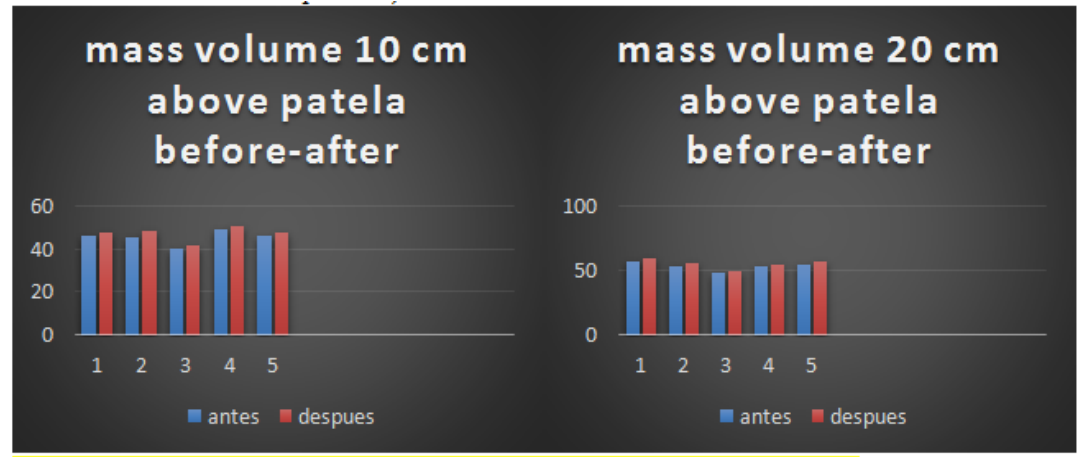

$1.94 \mathrm{~cm}$ average increase

$1.82 \mathrm{~cm}$ average increase

Table 6 Comparison between first evaluation and after 6 weeks of traditional resistance training (IRM evaluation test in quadriceps and hamstrings muscles)

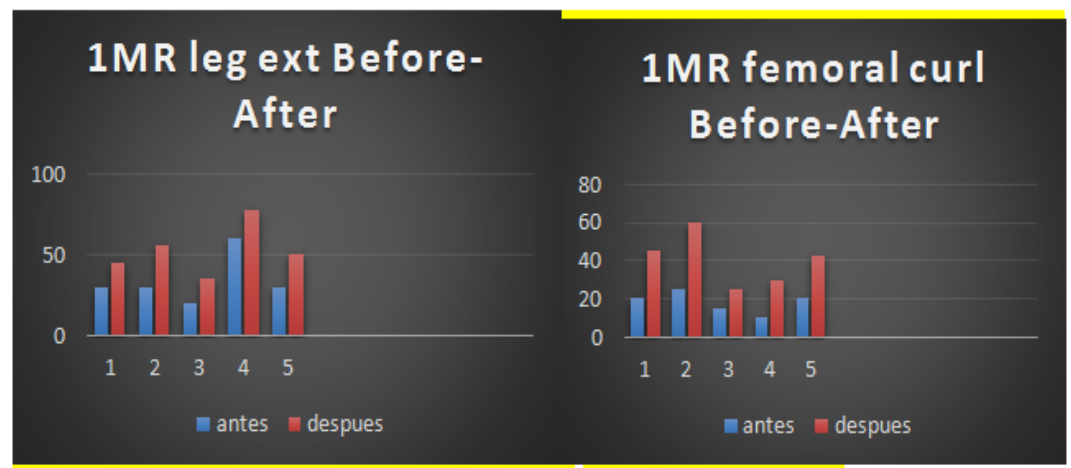

$18.71 \mathrm{~b}$ average increase in 1RM

$22.51 \mathrm{~b}$ average increase in $1 \mathrm{RM}$

\section{Data analysis}

Table 7-9

Table 7 Comparison table I and 4

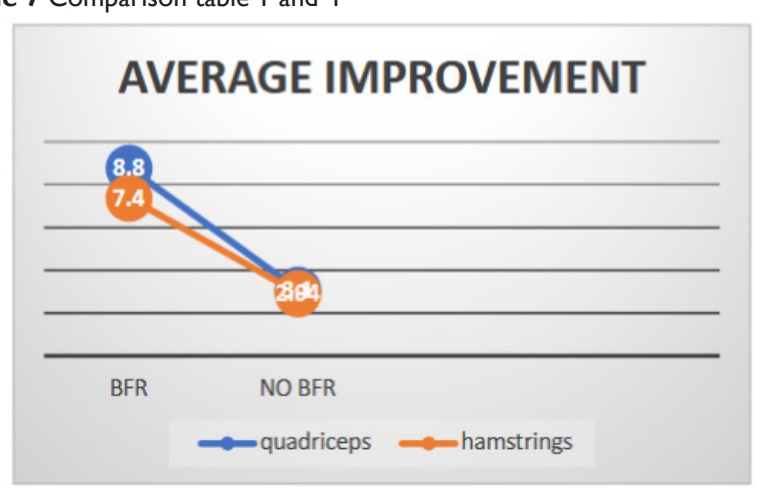

Table 8 Comparison table 2 and 5

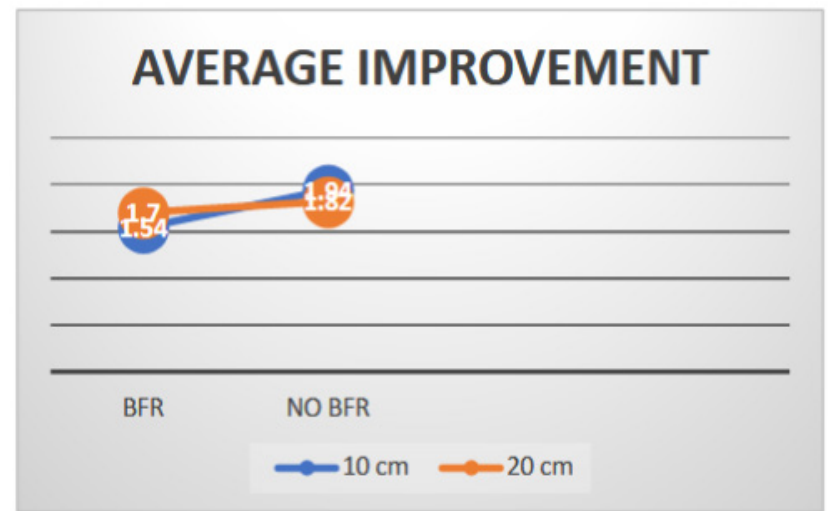


Table 9 Comparison table 3 and 6

\section{AVERAGE IMPROVEMENT}

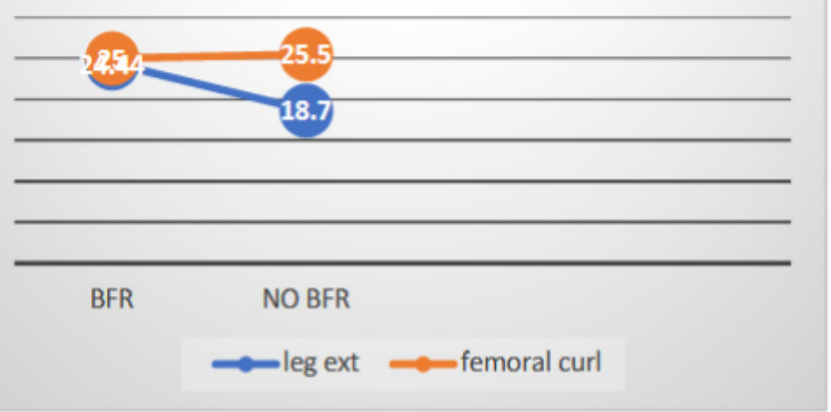

\section{Results}

At the end of the 6 weeks training all 10 individuals were evaluated with the same test from the beginning, no one dismissed intervention or follow-up procedures, both groups had improvements, with a greater improvement for BFR group in dynamometer and 1RM evaluation and greater improvement in mass volume measurement in control group, although these difference were not significant.

\section{Conclusion}

After the analysis, we conclude that BFR combined with low load resistance training is an effective method to treat the common consequences of ACL post-surgical procedures without the harms related to high intensity load training. We have also seen it is an effective way to decrease pain and instability sensation that often appear with high load resistance training after ACL surgery procedures.

\section{Limitations}

This study is limited by the small sample size and the limitation to have access to an ACL post-surgery population who met the inclusion criteria in a specific frame of time. The sample population in this study does not represent the general population, which may make it difficult to translate these findings to a more diverse population with another injuries or health issues involved, the compression level was applied homogeneously at $150 \mathrm{mmhg}$ in all participants without considering previous mass volume of each participant.

In addition, this study was conducted in a real clinical setting and not in a controlled lab. Further research is needed to determine if a higher volume of the population with a controlled lab evaluation and different compressions adapted to their initial stage Will produce the same positive effects. Patients should also be evaluated for long-term effects comparing both training methods.

\section{Acknowledgments}

Gratefulness to the team of Fisioandcare clinic for their kindly cooperation with the article data and development.

\section{Conflict of interest}

The author declares that there is no conflicts of interest.

\section{References}

1. Haruyasu Ohta, Hisashi Kurosawa, Hiroshi Ikeda, et al. Low-load resistance muscular training with moderate restriction of blood flow after anterior cruciate ligament reconstruction. Acta Orthopaedica Scandinavica. 2003;74(1):62-68.

2. Scott BR, Loenneke JP, Slattery KM, et al. Blood flow restricted exercise for athletes: A review of available evidence. J sci Med Sport. 2016;19(5):360-367.

3. Muhammad K, Hamed I. Moderate Blood Flow Restriction Training. MOJ Sports Med. 2017;1(3):00012.

4. Barenius B, Ponzer S, Shalabi A, et al. Increased risk of osteoarthritis after anterior cruciate ligament reconstruction: a 14-year follow-up study of a randomized controlled trial. Am J Sports Med. 2014;42(5):1049-1057.

5. Abe T, Kearns CF, Sato Y. Muscle size and strength are increased following walk training with restricted venous blood flow from the leg muscle, Kaatsu-walk training. J Appl Physiol (1985). 2006;100(5):14601466.

6. Wilson JM, Lowery RP, Joy JM, et al. Practical blood flow restriction training increases acute determinants of hypertrophy without increasing indices of muscle damage. J Strength Cond Res. 2013;27(11):3068-3075.

7. Abe T, Sakamaki M, Fujita S, et al. Effects of Low-Intensity Walk Training with Restricted Leg Blood Flow on Muscle Strength and Aerobic Capacity in Older Adults. J Geriatr Phys Ther. 2010;33(1):34-40.

8. Behringer M, Behlau D, Montag $\mathrm{J}$, et al. Low-intensity sprint training with blood flow restriction improves $100 \mathrm{~m}$ dash. J Strength Cond Res. 2017;31(9):2462-2472.

9. Pope, Willardson JM, Schoenfeld BJ. Exercise and blood flow restriction. J Strength Cond Res. 2013;27(10):2914-2926.

10. Sousa J, Neto GR, Santos HH, et al. Effects of strength training with blood flow restriction on torque, muscle activation and local muscular endurance in healthy subjects. Biol Sports. 2017;34(1):83-90.

11. Lowery RP, Joy JM, Loenneke JP, et al. Practical blood flow restriction training increases muscle hypertrophy during a periodized resistance training programme. Clin Physiol Funct Imaging. 2014;34(4):317-321.

12. Wilson JM, Lowery RP, Joy JM, et al. Practical blood flow restriction training increases acute determinants of hypertrophy without increasing indices of muscle damage. J Strength Cond Res. 2013;27(11):3068-3075.

13. Loenneke JP, Fahs CA, Rossow LM, et al. Blood flow restriction pressure recommendations: a tale of two cuffs. Front Physiol. 2013;4:249.

14. Karabulut M, Sherk VD, Bemben DA, et al. Inflammation marker, damage marker and anabolic hormone responses to resistance training with vascular restriction in older males. Clin Physiol Funct Imaging. 2013;33(5):393-399.

15. Behringer M, Behlau D, Montag J, et al. Low-intensity sprint training with blood flow restriction improves $100 \mathrm{~m}$ dash. J Strength Cond Res. 2017;31(9):2462-2472.

16. Owens Recovery Science. ACL+Blood Flow Restriction Part 2. 